\title{
Dynamical properties of the delta-kicked harmonic oscillator
}

\author{
G. A. Kells, ${ }^{*}$ J. Twamley, and D. M. Heffernan \\ Department of Mathematical Physics, National University of Ireland, Maynooth, Ireland
}

(Received 31 July 2003; published 27 July 2004)

\begin{abstract}
We propose an efficient procedure for numerically evolving the quantum dynamics of delta-kicked harmonic oscillator. The method allows for longer and more accurate simulations of the system as well as a simple procedure for calculating the system's Floquet eigenstates and quasienergies. The method is used to examine the dynamical behavior of the system in cases where the ratio of the kicking frequency to the system's natural frequency is both rational and irrational.
\end{abstract}

DOI: 10.1103/PhysRevE.70.015203

PACS number(s): 05.45.Pq, 03.65.-w, 05.45.Mt

Kicked Hamiltonian systems are firmly established as prototypical models for studying chaos. Enormous effort has been spent examining models such as the standard map and the kicked rotator. The study of kicked degenerate systems, which do not obey the Kolmogorov-Arnol'd-Moser theorem, has received relatively little attention but displays fascinating dynamical properties both classically and quantum mechanically. The kicked harmonic oscillator (KHO) is an example of such a system. The system was originally proposed as a two-dimensional model of kicked charges in a uniform magnetic field [1]. It has also been proposed as a model for electronic transport in semiconductor superlattices [2] and atom optic modeling using ion traps [3]. In particular, the quantum kicked harmonic oscillator, in the same way as the quantum kicked rotator [4], may be simulated efficiently on a quantum information processor using the quantum fractional Fourier transform [5].

The Hamiltonian of the system may be written as

$$
H_{K H O}=\omega_{H O}\left(\frac{p^{2}}{2}+\frac{q^{2}}{2}\right)+V(q) \sum_{n=-\infty}^{\infty} \delta\left(t-n T_{K}\right)
$$

The kicking potential $V(q)$ in our system is given by

$$
V(q)=\mu \cos (k q)
$$

where $k$ is a parameter related to $\omega_{H O}$ and the mass $m$. It is distinct from other widely studied systems because of the presence of two different frequencies in the Hamiltonian, namely, the natural frequency of the harmonic oscillator, $\omega_{H O}$, and the kicking frequency $\omega_{K}=2 \pi / T_{K}$. It is the ratio between these frequencies, $\omega_{H O} / \omega_{K}=1 / R$, and the strength of the kicking potential that determines the global behavior of the system [1]. It is appropriate to note here that the kicking potential we use is even. Using an odd kicking potential, say $V(q)=\mu \sin (k q)$, changes the global behavior of the system dramatically [6]. We do not attempt to analyze this case although the numerical techniques that we present later can be applied to any delta-kicking potential.

\footnotetext{
*Electronic address: gkells@thphys.may.ie
}

Understanding the quantum system analytically has proved to be problematic. While expressions have been found for the Floquet operator [7], these generally involve complicated infinite summations of Bessel functions and are too cumbersome to yield any important information about the dynamics of the system. Numerical simulations are therefore essential to understand how the system evolves. However, in general, factors such as memory and computation time reduce the accuracy of simulations before much can be concluded about the eigensolutions or long time dynamical evolution of the system. We outline a procedure, based on the fractional Fourier transform, that allows for efficient numerical simulation of the system's evolution as well as an effective and straightforward approach to calculate the system's Floquet operator. We use this approach to confirm previous results for the system where $R=4,5$ as well as show that delocalization can occur when $R$ is irrational if the kicking potential is strong enough.

The Floquet operator for this system can be factorized into two parts:

$$
U_{K H O}\left(T_{K}\right)=U_{S H O}\left(T_{K}\right) U_{k i c k},
$$

where because of the $\delta$ function in Eq. (1) we can express the kick operator as

$$
U_{k i c k}=\exp [(-i / \hbar) \mu \cos (k q)]
$$

which is clearly diagonal in the position basis and between kicks

$$
U_{S H O}\left(T_{K}\right)=\exp \left(\left[-i \omega_{H O} T_{K}\left(p^{2}+q^{2}\right)\right] / 2 \hbar\right)
$$

which is diagonal in the Fock state basis but not in the position basis or the momentum basis. Changing from the number basis to the position basis requires a matrix-vector multiplication ( $N^{2}$ operations) where $N$ is the number of dimensions being used to approximate the infinite dimensional Hilbert space. However, it is well known that changing between the position and momentum bases can be done through Fourier transforms. This fact is used to great advantage in the split step method [8]. In this method the exact operator $U_{S H O}$, applied for the small duration $\Delta t$, can be approximated by 


$$
\begin{aligned}
U_{S H O}^{\prime}(\Delta t)= & \exp \left(\frac{-i \omega_{H O} \Delta t p^{2}}{4 \hbar}\right) \exp \left(\frac{-i \omega_{H O} \Delta t q^{2}}{2 \hbar}\right) \\
& \times \exp \left(\frac{-i \omega_{H O} \Delta t p^{2}}{4 \hbar}\right)
\end{aligned}
$$

where we use the fast Fourier transform (FFT) [9] to perform the change of basis. Although this method is not exact, the error is $O(\Delta t)^{3}$, and can therefore be made acceptably small by an appropriate choice of $\Delta t$. The number of operations needed to evolve the system from time $t$ to time $t+\Delta t$ is $O(N \ln N)$. However, using the stability condition for the split-step method [10], $\Delta t<l^{2} / \pi$, where $l$ is the grid spacing, we roughly estimate the number of operations needed to accurately evolve the system over a finite time to be of $O\left(N^{2} \ln N\right)$. The essential point is that we avoid the use of matrix-vector multiplications through the use of fast Fourier transforms. This technique is relatively efficient computationally and does not require large amounts of storage.

It is possible, however, to exploit certain symmetries in the Hamiltonian to evolve the system with far greater efficiency. The previous discussion regarded the Fourier transform as a passive transformation, that is, one that changes the position basis to the momentum basis. However, for the harmonic oscillator, a Fourier transform can also be regarded as an active transformation. In 1980, Namias defined the fractional Fourier transform in terms of the Hermite polynomials and showed that it is in fact the time evolution operator of the harmonic oscillator up to a phase [11]. Explicitly, taking $\Psi(x, t)$ as the time-dependent wave function of the harmonic oscillator, he showed that

$$
\Psi(x, t)=U_{S H O}(t) \Psi(x, 0)=e^{-i t / 2} F_{-t} \Psi(x, 0),
$$

where the operator $F_{-t}$ is a generalization of the ordinary Fourier transform [12]. Indeed the latter is just the special case when $t=\pi / 2$. This result is important for our system because of the existence of fast fractional Fourier transforms (FFrFT's) [13]. There are a number of algorithms in the literature for performing the fast fractional Fourier transform as efficiently as the regular FFT. The basic idea is to factorize the FrFT into a series of simpler transformations. These transformations may include the ordinary FFT, scaling operations, and what are known in the signal processing field as chirp convolutions and multiplications [14]. A selection of web based resources can be found in Ref. [15] and for a comprehensive review of the fractional Fourier transform see Ref. [16].

What all this means is that, in the position basis, the evolution of the wave function over a finite time may be written as

$$
\Psi\left(x, T_{K}\right) \equiv U_{K H O}\left(T_{K}\right) \Psi(x, 0)=e^{-i V(x) / \hbar} e^{-i T_{K} / 2} F_{-T_{K}} \Psi(x, 0),
$$

and can be performed in about $O(N \ln N)$ operations. This allows us to perform highly efficient numerical simulations of pure state quantum dynamics in Hilbert space dimensions of about $2^{17}$. This enables us to follow the diffusing quantum evolution for larger times and in greater detail. Simulations of such a size would be prohibitively costly in memory and processor time using other simulation techniques.

As an additional advantage, the Floquet matrix itself can be calculated. This is a consequence of Eq. (8) and the fact that the fractional Fourier transform matrix can be generated without much additional overhead [13]. This allows for quick and straightforward calculation of the Floquet eigenstates and eigenvalues using the standard LAPACK routines.

One of the interesting properties of the system given in Eqs. (1) and (2) is the presence of a stochastic web that spans all of the classical phase space for certain values of the parameter $R$. Indeed the values of $R$ for which this crystalline structure appears can be seen to be related to the tessellation of the phase plane, where the values of $R=3,4,6$ represent filling (tiling) the plane with triangles, squares, or hexagons, respectively (we ignore the degenerate cases of $R=1,2$ ). In the quantum mechanical problem these specific values of $R$ have, for all values of the kicking strength, been shown to give rise to extended quantum eigenstates and therefore delocalization of the quantum state over time [17-19].

In the other cases where $R$ is an integer, a quasicrystalline structure is observed in the classical phase plane. In the quantum system the rate of energy growth is largely suppressed $[18,20]$. These results do not appear to contradict those of the tight binding prediction [19], where extended states are predicted for all rational values of the frequency ratio. Indeed, there is a value of the kick strength, above which the quantum system diffuses at a rate less than, but comparable to, that of the classical system [20], whose energy is known to grow linearly with time.

In the case of irrational frequency ratios all traces of a crystalline structure disappear in the classical phase plane. The tight binding model predicts localization for all values of the kick strength [19], and this has been supported numerically for low kick strengths [18]. As far as we are aware there is no published numerical data for irrational frequency ratios for this system with large kick strength.

To study the localization properties of the system we first investigate the mean energy after each application of the Floquet operator. We calculate

$$
E(t)=\left\langle\psi_{t}\left|\left(p^{2} / 2\right)+\left(q^{2} / 2\right)\right| \psi_{t}\right\rangle
$$

as a function of the discrete time $T_{K}$ or the kick number $n$. A direct comparison may be made between the quantum state and a classical ensemble. Defining $\alpha=(q+i p) / \sqrt{2 \hbar}$ we have the wave function of a coherent state centered at the coordinates $(q, p)$,

$$
\psi(q)=\left(\frac{\omega_{0}}{\pi \hbar}\right)^{1 / 4} \exp \left(-\frac{\omega_{0}}{2 \hbar} q^{2}+\sqrt{\frac{2 \omega_{0}}{\hbar}} \alpha q-\frac{|\alpha|^{2}}{2}-\frac{\alpha^{2}}{2}\right) .
$$

The Wigner function of this state is a two-dimensional (2D) Gaussian that is centered about the point $(q, p)$ [21]. The Husimi function is very similar to this and can be regarded as the convolution of the Wigner function with a 2D Gaussian centered at the origin. In the numerical simulations we compare the quantum evolution of a coherent state with the classical evolution of an ensemble of normally distributed particles initially centered around $(q, p)$ and possessing the same marginals as the corresponding Husimi function. 

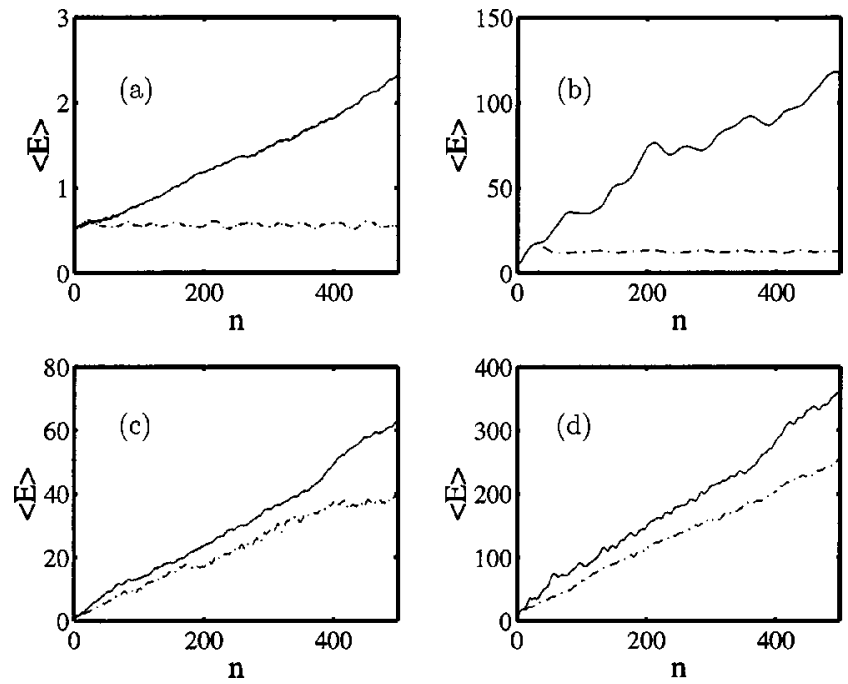

FIG. 1. Mean energy vs time for $\omega_{H O} / \omega_{K}=1 / R=1 / 4$ and $\hbar=1$. (a, b) $\mu=0.5$ with initial state centered at $(0,0)$ and $(0, \pi)$, respectively. (c, d) $\mu=2$ with initial state centered at $(0,0)$ and $(0, \pi)$, respectively. A Hilbert space of dimension of $2^{16}$ was used in the simulation. Solid (dashed) curves represent the quantum (classical) system.

When $R=4$, the quantum system can be shown analytically to be identical to the well-studied kicked Harper model [6]. In this case the quantum system diffuses for all values of the kick strength $\mu$ and for all initial conditions. To demonstrate this we center the initial state on a classical stable point $(q, p)=(0,0)$, and note that the energy of the quantum state grows with time (or kick number $n$ ), while the energy of the classical state remains bounded. From Fig. 1(a), we see that the quantum system is able to tunnel through the separatrix which completely isolates classical trajectories. For completeness we also illustrate how both the quantum and classical systems diffuse when placed on an unstable point $(q, p)=(0, \pi)$ on the classical system's stochastic web. It is worth noting that at $\mu=0.5$, the stochastic web is too small to allow classical diffusion. However we see that the quantum system uses the separatrix to significantly increase its rate of diffusion.

In the case where $R=5$, the classical phase plane shows a quasicrystalline structure. From Fig. 2 we can see that at low kicking strengths diffusion of the quantum system is somewhat suppressed. However, above a certain critical value of the kick strength $\mu$, which depends on the initial position of the quantum state, this suppression no longer seems to exist.

We see a similar type of global behavior when the frequency ratio is irrational even though the structures of the phase space patterns are quite different. Both the quantum and classical systems remain bounded up to different critical values of the kick strength $\mu$ (again the precise value of this parameter depends on the initial conditions). Above these values the system begins to diffuse linearly. In the same way as before, there exists a range of kick strengths where the classical system diffuses and the quantum motion remains suppressed; see Figs. 3(b) and 3(c). It would appear that, in this region, the quantum state cannot flow along the same chaotic trajectories as the classical ensemble. This is oppo-
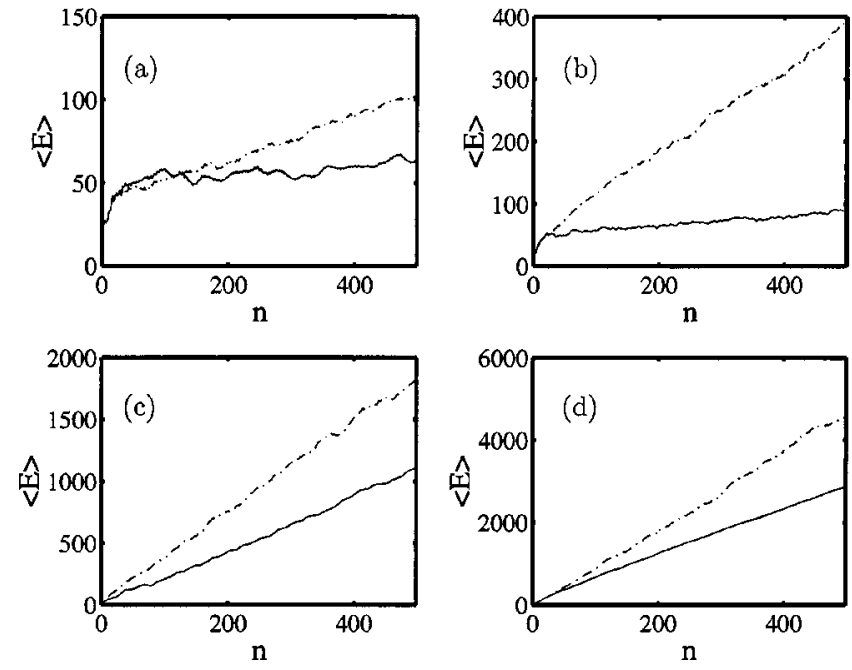

FIG. 2. Mean energy vs time for $\omega_{H O} / \omega_{K}=1 / R=1 / 5$ and $\hbar=1$. (a) $\mu=1$, (b) $\mu=2$, (c) $\mu=4$, and (d) $\mu=6$. The initial state was centered at $(q, p)=(7.5,0)$. A Hilbert space of dimension of $2^{16}$ was used in this simulation. Solid (dashed) curves represent the quantum (classical) system.

site to the case for which $R=4$, where at low kick strengths, the quantum system appears to exploit the infinitesimal stochastic web to aid its dispersion; see Figs. 1(a) and 1(b).

As we have mentioned, using the definition of the Floquet operator given in Eq. (8), we can easily find a finite dimensional matrix that can then be diagonalized by brute force. This method has allowed us to find eigenvalues and eigenvectors of matrices up to $3000 \times 3000$. To go larger requires more random access memory. This can then be used to examine the structure of these eigenstates in detail as well as examine the system's eigenvalue statistics. As an example we show that, in the case of irrational kick frequencies, the Floquet operator contains some eigenstates that are clearly
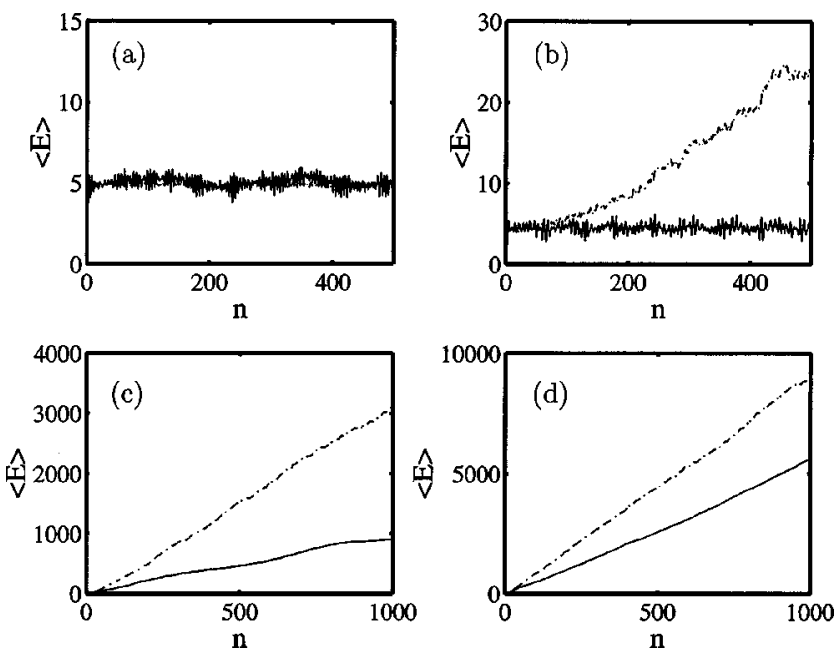

FIG. 3. Mean energy vs time for $\omega_{H O} / \omega_{K}=1 / R=(\sqrt{5}+1) / 2$ and $\hbar=1$. (a) $\mu=1$, (b) $\mu=2$, (c) $\mu=4$, and (d) $\mu=6$. The initial state was centered at $(q, p)=(\pi, 0)$. A Hilbert space of dimension of $2^{17}$ was used for this simulation. Solid (dashed) curves represent the quantum (classical) system. 

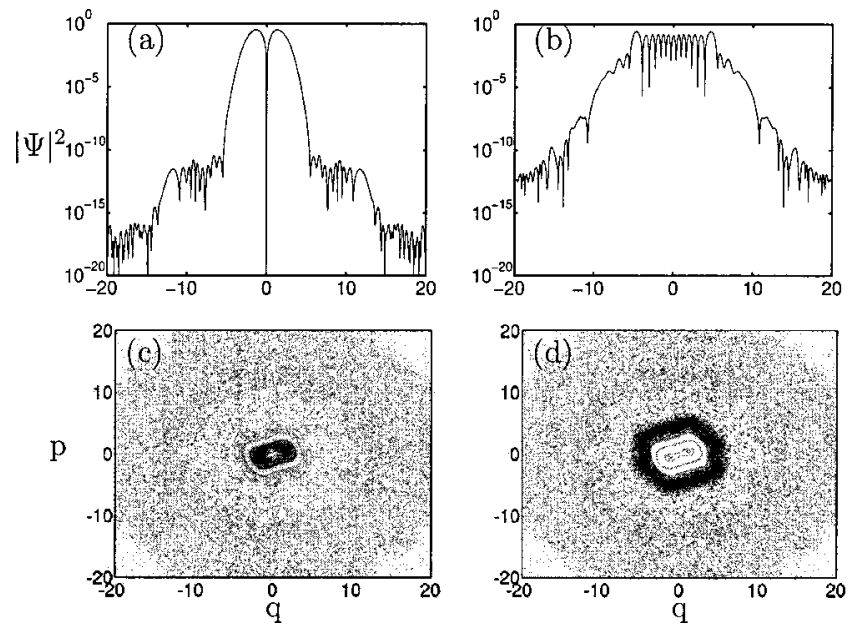

FIG. 4. (a,b) Low energy eigenstates for the irrational system with kick strength $\mu=1$, plotted on a logarithmic scale. Below each state, (c) and (d), are their Husimi functions plotted in overlay with the classical phase space Poincaré plot. A Hilbert space of dimension of $2^{10}$ was used for these simulations.

localized in space and some that appear to be extended. The existence of these states highlights what we have already found in the diffusion simulations. At low kick strength (say $\mu=1$ ), Fig. 4, an initial state may have eigenstate components which are localized. This is not the case, however, if we examine the eigenstates for the system with a larger kick strength. When $\mu=6$ even the lower energy eigenstates appear to have some sort of extended nature (Fig. 5). These figures do not show all the individual eigenstates. We have zoomed in on a small section to emphasize the Husimi function's structural similarity to classical phase map.

In conclusion, we have addressed the numerical problems associated with studying the quantum delta-kicked harmonic
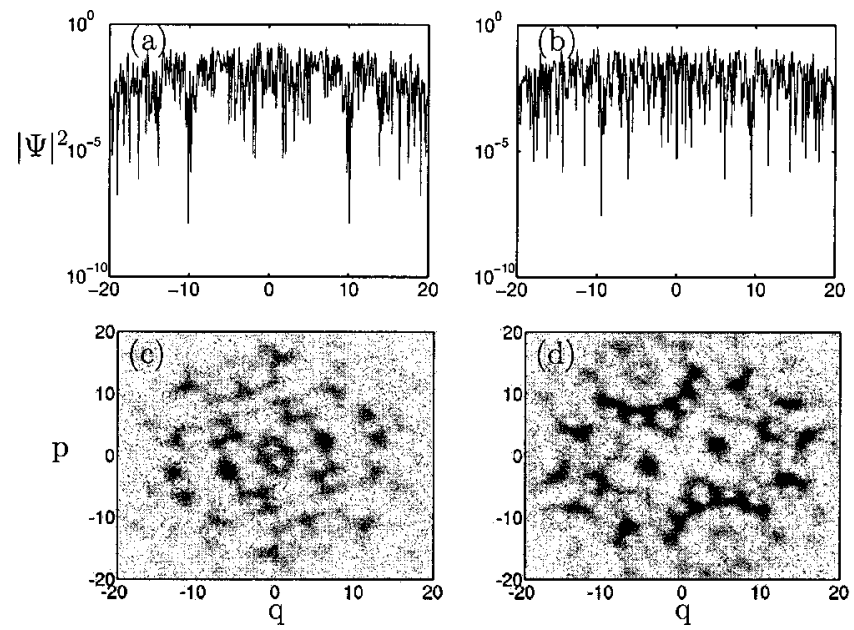

FIG. 5. (a,b) Low energy eigenstates for the irrational system with kick strength $\mu=6$, plotted on a logarithmic scale. Below each state, (c) and (d), are their Husimi functions plotted in overlay with the classical phase space Poincaré plot. A Hilbert space of dimension of $2^{10}$ was used for these simulations.

oscillator. We have shown that the fractional Fourier transform may be used to infer crucial numerical information about the system. This includes improving the speed and accuracy with which we can evolve the system as well as giving an easy and effective way to analyze the system's Floquet eigenstates and quasienergies. This improved numerical technique will assist in future studies of this system dynamics using either a quantum information processor or the various physical systems mentioned in the Introduction.

This research was supported by funding from Enterprise Ireland under research grant no. EI SC/2001/059.
[1] G. M. Zaslavsky et al., Zh. Eksp. Teor. Fiz. 91, 500 (1986) [Sov. Phys. JETP 64, 294 (1986)]; A. A. Chernikov et al., Physica D 33, 65 (1988)]; G. M. Zaslavsky et al., Weak Chaos and Quasi-Regular Patterns (Cambridge University Press, New York, 1991); A. J. Lichtenberg and B. P. Wood, Phys. Rev. A 39, 2153 (1989); I. Dana and M. Amit, Phys. Rev. E 51, R2731 (1995), and references therein.

[2] T. M. Fromhold et al., Phys. Rev. Lett. 87, 046803 (2001).

[3] S. A. Gardiner et al., Phys. Rev. Lett. 79, 4790 (1997).

[4] B. Levi et al., Phys. Rev. E 67, 046220 (2003).

[5] A. Klappenecker and M. Rötteler, Phys. Rev. A 67, 010302 (2003).

[6] I. Dana, Phys. Rev. Lett. 73, 1609 (1994).

[7] M. V. Daly and D. M. Heffernan, Acta Phys. Pol. A 89, 5 (1996).

[8] M. D. Feit et al., J. Comput. Phys. 47, 412 (1982).

[9] J. W. Cooley and J. W. Tukey, Math. Comput. 19, 297 (1965).

[10] J. A. C. Weideman and B. M. Herbst, SIAM (Soc. Ind. Appl. Math.) J. Numer. Anal. 23, 485 (1986).

[11] V. Namias, J. Inst. Math. Appl. 25, 241 (1980).
[12] Namias defines the Fourier transform as $F_{\pi / 2} f(x)$ $=1 / \sqrt{2 \pi} \int f\left(x^{\prime}\right) \exp \left(i x x^{\prime}\right) d x^{\prime}$. Many people call this the inverse Fourier transform.

[13] H. M. Ozaktas et al., IEEE Trans. Signal Process. 44, 2141 (1996); H. M. Ozaktas et al., The Fractional Fourier Transform with Applications in Optics and Signal Processing (Wiley, New York, 2000).

[14] The code used was taken from the site http:// www.ee.bilkent.edu.tr/ haldun/wileybook.html which is based upon the routines given in Ref. [13].

[15] See Dr. YangQuan Chen's FRFT Webpages http:// mechatronics.ece.usu.edu/foc/FRFT/

[16] A. Bultheel and H. Martínez, Department of Computer Science, Katholieke Universiteit Leuven, Report No. TW337, 2002 (unpublished).

[17] G. P. Berman et al., Nonlinearity 4, 543 (1991).

[18] F. Borgonovi and L. Rebuzzini, Phys. Rev. E 52, 2302 (1995).

[19] M. Frasca, Phys. Lett. A 231, 344 (1997).

[20] D. Shepelyansky and C. Sire, Europhys. Lett. 20, 95 (1992).

[21] M. Hillery et al., Phys. Rep. 106, 121 (1984). 